\title{
Neuropsychological functioning, illness perception, mood and quality of life in chronic fatigue syndrome, autoimmune thyroid disease and healthy participants
}

\author{
A. Dickson ${ }^{1 *}$, A. Toft ${ }^{2}$ and R. E. $\mathrm{O}^{\prime}$ Carroll $^{3}$ \\ ${ }^{1}$ School of Health and Social Sciences, Napier University, Edinburgh, UK \\ ${ }^{2}$ Department of Endocrinology, Royal Infirmary of Edinburgh, Edinburgh, UK \\ ${ }^{3}$ Department of Psychology, University of Stirling, Stirling, UK
}

Background. This study attempted to longitudinally investigate neuropsychological function, illness representations, self-esteem, mood and quality of life (QoL) in individuals with chronic fatigue syndrome (CFS) and compared them with both healthy participants and a clinical comparison group of individuals with autoimmune thyroid disease (AITD).

Method. Neuropsychological evaluation was administered at two time points, five weeks apart. Twenty-one individuals with CFS, 20 individuals with AITD and 21 healthy participants were matched for age, pre-morbid intelligence, education level and socio-economic status (SES). All groups also completed measures of illness perceptions, mood, self-esteem and QoL at both time points.

Results. The CFS group showed significantly greater impairment on measures of immediate and delayed memory, attention and visuo-constructional ability, and reported significantly higher levels of anxiety and depression. After controlling for the effects of mood, the CFS group still demonstrated significant impairment in attention. The CFS group also reported significantly lower self-reported QoL than the AITD and healthy participants. In terms of illness perceptions, the AITD group believed that their condition would last longer, that they had more treatment control over their condition, and reported less concern than the CFS group.

Conclusions. These results suggest that the primary cognitive impairment in CFS is attention and that this is not secondary to affective status. The lower treatment control perceptions and greater illness concerns that CFS patients report may be causally related to their affective status.

Received 19 December 2007; Revised 22 October 2008; Accepted 21 November 2008; First published online 15 January 2009

Key words: Attention, cognitive impairment, depression, illness perception, memory, quality of life.

\section{Introduction}

Chronic fatigue syndrome (CFS) is a condition characterized by persistent, disabling fatigue lasting for 6 months or more (Fukuda et al. 1994). In addition to fatigue, individuals with the condition often experience a combination of chronic and concurrent somatic symptoms (e.g. headache, sore throat, swollen glands and myalgia) and a host of cognitive deficits (e.g. impaired concentration, attention and memory and slowed thinking). Prevalence of the condition is estimated at approximately $0.3-0.6 \%$ of the population (Campion et al. 1998) and CFS is most commonly

* Address for correspondence: Dr A. Dickson, School of Health and Social Sciences, Napier University, Edinburgh EH10 5LG, UK.

(Email: a.dickson@napier.ac.uk) found in females between the ages of 20 and 40 years (Pheby, 1999).

A distinct focus of the CFS literature has been cognitive function (Komaroff, 1993; Ray et al. 1993; Krupp et al. 1994; DeLuca et al. 1995, 2004; Marshall et al. 1997; Wearden \& Appleby, 1997; Christodoulou et al. 1998; Michiels et al. 1999; Ross et al. 2001; Lange et al. 2005). However, variations in methodological approaches, participant selection criteria and statistical analyses make direct comparisons between these studies difficult. Indeed, such methodological variations may account for the inconsistencies in the research findings to date. For example, some authors have highlighted that CFS patients tend to perform within the normal range on most tests (Grafman et al. 1993; Sandman et al. 1993; Cope et al. 1995), others have reported overall slight impairment (Krupp et al. 
1994; DeLuca et al. 1995), and others have shown that CFS patients almost always show slowed motor or cognitive processing abilities (Ray et al. 1993; Krupp et al. 1994; DeLuca et al. 1995), and when compared to healthy controls, CFS patients are more likely to show neuropsychological impairments in attention, motor speed, memory and speed of information processing (Marshall et al. 1997; Wearden \& Appleby, 1997; Johnson et al. 1998; Busichio et al. 2004; DeLuca et al. 2004). It has also been claimed that impairments in cognitive functioning may be a partial consequence of affective status (Cope et al. 1995; DeLuca et al. 1995; Wearden \& Appleby, 1997); CFS patients who also have co-morbid depression show significantly poorer performances on cognitive assessments than their non-depressed counterparts (Marshall et al. 1997; Wearden \& Appleby, 1997).

There is often a disparity between the degree of subjective complaints of cognitive impairment in CFS patients and the degree of that cognitive impairment on neuropsychological examination (Ray et al. 1993; Wood et al. 1994). Explanations for this disparity include the notion that CFS patients may be able to perform well at the time of assessment but this may ultimately come at the cost of subsequent increased fatigue (Ray et al. 1993); the idea that levels of arousal and motivation are somewhat different in a laboratory environment to that of everyday life; and the notion that patients may exaggerate their reported cognitive impairment due to depressed mood (Wearden \& Appleby, 1997).

The relationship between CFS and depression is a complex one. A large proportion of the CFS population are depressed (David, 1991; Van Hoof et al. 2003) and prevalence rates vary between $50 \%$ and $60 \%$ (Ax et al. 2001). When comparisons are made to other chronic illness populations, such as multiple sclerosis and rheumatoid arthritis (RA), CFS groups report increased rates of depression (Pepper et al. 1993; Wood et al. 1994; Johnson et al. 1996). Proposed explanations for the relationship between CFS and depression include: (1) major depression (MD) as the primary cause of CFS; (2) MD as a secondary condition to CFS; and (3) MD and CFS exist as co-morbid conditions. Again, there are inconsistencies in the literature. For example, although some researchers have argued that CFS is an atypical presentation of primary MD, anxiety or somatization disorder (Katon et al. 1991; Cope et al. 1996; Manu et al. 1998; van der Linden et al. 1999; Wessely et al. 1999; Wessely \& White, 2004), others have proposed that CFS is a distinct medical illness caused primarily by immune system dysfunction (Hickie et al. 1990; Komaroff \& Buschwald, 1991). CFS patients often claim that cognitive impairment is one of the main factors contributing to impaired social, relationship and occupational dysfunction (Abbey \& Garfinkel, 1990; Ax et al. 2001). This could suggest that depression may be secondary to CFS (MacDonald et al. 1996; Ax et al. 2001).

Illness perceptions may play a central role in perpetuating disability levels in CFS (Surawy et al. 1995; Chalder et al. 1999; Edwards et al. 2001; Moss-Morris $\&$ Petrie, 2001). More specifically, catastrophic thinking has been found to be related to both disability and fatigue (Petrie et al. 1995) and aspects of illness beliefs have been found to predict the progression of CFS (e.g. increased perceptions of controllability of the condition tend to be linked to a better outcome) (Ray et al. 1997). On the whole, CFS groups have been found to report increased illness identities, increased perceptions of the seriousness of their condition and are more likely to attribute their condition to immune system dysfunction than comparison groups (e.g. RA) (MossMorris \& Chalder, 2003). These illness beliefs may be causally related to role and social dysfunction in CFS (Leventhal et al. 1989; Petrie et al. 1995; Surawy et al. 1995; Ray et al. 1997; Chalder et al. 1999; Edwards et al. 2001; Moss-Morris \& Petrie, 2001; Moss-Morris \& Chalder, 2003).

A further focus of CFS literature to date has been on quality of life (QoL). Despite inconsistency in defining QoL and the various domains measured, findings have been relatively consistent. For example, both subjective QoL and health-related QoL have been found to be low in the CFS population when compared to other groups (Hardt et al. 2001; Rakib et al. 2005). More specifically, Anderson \& Ferrans (1997) reported that QoL is particularly and uniquely disrupted in CFS whereas Schweitzer et al. (1995) reported that social functioning (in terms of a loss of role functioning and social isolation) is particularly impaired.

Although previous research has played an important role in raising the profile of CFS, it has been often been criticized for failing to include an appropriate clinical comparison group (DeLuca, 1995). In response, the present study included a comparison group of individuals with autoimmune thyroid disease (AITD) and a healthy control group (matched for age, and social and educational background). AITD patients were selected as they share many similar symptoms to those experienced by CFS patients, particularly fatigue, low mood and subjective complaints of cognitive impairment. Findings from objective neuropsychological assessment in AITD are rarely reported. Longitudinal research assessing neuropsychological functioning in CFS is also rare. This study, therefore, aimed to compare neuropsychological functioning in these two groups in comparison 
with healthy individuals. We also aimed to investigate the relationship between impairment, illness perceptions, mood and QoL to better understand the interplay between these domains. Although previous research has generally investigated cognitive function cross-sectionally in CFS at one time point, the present research adopted a repeated-measures design to explore changes in cognitive functioning over time. This research is therefore both novel and timely in furthering our knowledge of cognitive function in CFS.

\section{Method}

\section{CFS participants: sampling and procedure}

UK National Health Service (NHS) ethical approval was sought from the South Glasgow and Clyde Local Research Ethics Committee, the Lothian Ethics Committee and from the University of Stirling. Twenty-one individuals with CFS were recruited by a lead CFS consultant [then based in the Southern General Hospital (SGH), Glasgow] $(n=16)$ and through a CFS support group $(n=5)$. Information letters explicitly detailing the nature of the research and the participant's involvement were sent to the lead CFS consultant (by post) and to the group leader at the CFS support group (by email). Forty-three letters were distributed by the SGH and 12 emails were sent by the CFS support group. The information letters also provided the first author's contact details. In this way, only those individuals who elected to take part in the research were involved. Once the participants had indicated a willingness to participate, the first author contacted them by telephone or email to arrange for the research to commence. The hospital referrals were assessed in a private consulting room in the SGH and the support group patients were assessed at the host institution.

Participants (11 males and 10 females) were aged between 18 and 69 years (mean 46.7 years, S.D. $=11.58$ ). Participants' estimated pre-morbid IQs varied from 97 to 116 (mean 108.1, S.D. =5.06) and they had spent between 10 and 19 years in full-time education (mean 14.9 years, S.D. $=2.72$ ). All participants were from the central belt of Scotland and had all been diagnosed by their general practitioner or consultant as having CFS. All participants fulfilled the Centers for Disease Control (CDC) diagnostic criteria for CFS (Fukuda et al. 1994). Six (three males and three females) of the 21 participants were taking prescribed medications at the time of assessment. These medications included cipralil $(10 \mathrm{mg})(n=1)$; prozac $(40 \mathrm{mg})(n=2)$; perindopril $(6 \mathrm{mg})(n=1)$; a combination of fluoxetine $(40 \mathrm{mg})$, trazadone $(150 \mathrm{mg})$, atravent and lorazepam $(1 \mathrm{mg})(n=1)$; and a combination of clonazepam, baclofen $(40 \mathrm{mg})$ and trimipramine $(250 \mathrm{mg})(n=1)$. We found no differences on any of the dependent variables when we compared the patients taking medication with the other CFS patients.

\section{AITD participants: sampling and procedure}

Twenty-one individuals with confirmed AITD (see below) who were complaining of symptoms consistent with hypothyroidism (e.g. tiredness, weight gain and low mood) but who were clinically euthyroid (whether taking thyroid supplements or not) were recruited by the lead endocrine consultant in the Royal Infirmary of Edinburgh (RIE). Appointment letters were posted to 22 patients with a date for the research to commence. All but one patient agreed to attend. The participants were assessed in a private consulting room in the RIE. One participant subsequently received a diagnosis of hypopituitarism and was thus excluded from the data set. The final sample therefore comprised 20 AITD individuals.

The 20 participants (three males and 17 females) were aged between 20 and 65 years (mean 43.5 years, S.D. =13.43). Participants' estimated pre-morbid IQs varied from 99 to 116 (mean 110, S.D. $=5.44$ ) and they had spent between 10 and 20 years in education (mean 14.9 years, S.D. $=3.29$ ). All participants were from the east of Scotland and varied in socio-economic status (SES).

Of the final sample, seven individuals (one male, six females) had Hashimoto's thyroiditis (HT). The diagnosis of HT was based on the finding of a typical goitre in the presence of antibodies directed against thyroid peroxidase. There were nine patients (one male, eight females) with spontaneous primary atrophic hypothyroidism (SPAH) and three patients (one male, two females) in whom thyroid failure developed following iodine-131 therapy for hyperthyroidism due to Grave's disease (GD). At the time of neuropsychological testing, all patients were clinically euthyroid with normal concentrations of serum free thyroxine and total triiodothyronine. Serum thyroid-stimulating hormone (TSH) was either normal or suppressed in all but one patient; that patient with untreated HT had a marginally elevated serum TSH of $5.7 \mathrm{mU} / 1$. Finally, all but two patients were currently taking thyroxine (ranging from $50 \mu \mathrm{g}$ to $200 \mu \mathrm{g}$ daily) at the time of assessment. We found no differences on any of the dependent variables when we compared the patients with spontaneous primary atrophic hypothyroidism versus the other thyroid patients.

\section{Healthy controls: sampling and procedure}

Twenty-one lecturers and evening-class students at a Glasgow Further Education (FE) college were 
recruited. Written permission was sought from the Assistant Principle at the FE college for the first author to approach students during their evening classes. The first author gave a short presentation to three groups of students (sports studies, nail care and beauty care) detailing the nature of the research and explicitly highlighting what would be expected of the participants. Willing students were invited to participate that evening. Lecturers were recruited from the staff-room, were informed of the nature of the research and their involvement in it and again were asked to participate. All willing participants were assessed in a private interviewing room within the college.

Participants were aged between 24 and 57 years (mean 39.5 years, S.D. $=10.64$ ); there were six males and 15 females. Participants were lecturers $(n=6)$ and students $(n=15)$ and were from the central belt of Scotland. The participants' estimated IQs varied from 102 to 115 (mean 108.1, S.D.=4.05) and they had spent between 12 and 19 years in education (mean 14.5 years, S.D. $=1.91$ ). All of the participants varied in socio-economic background. Participants self-reported as being 'healthy'; they had no medical complaints, prior or current psychiatric disorder and were taking no prescribed medications at the time of assessment.

\section{Measures}

All participants were instructed to read a participant information sheet detailing the nature of the research and the participant's involvement. All participants subsequently signed a consent form and gave basic demographic details such as age, occupation, number of years in education and postcode. All participants then completed the following:

Wechsler Test of Adult Reading (Wechsler, 1981). This test estimates pre-morbid intellectual functioning and involves presenting the participant with a card showing 50 words. Participants pronounce the words as correctly as possible. The higher the score, the higher the participants' estimated pre-morbid level of intellectual functioning.

Repeatable Battery for the Assessment of Neuropsychological Status (RBANS; Randolph, 2002). Five domains are assessed: (1) immediate memory; (2) visuospatial/ constructional ability; (3) language; (4) attention; and (5) delayed memory. Tests include list learning (recall), story memory, figure copy, line orientation, picture naming, semantic fluency, digit span, and coding. The attention domain score is a composite score based on performance on the digit span and coding tests. There are two matched, parallel versions of the RBANS: version A is used at time 1 (T1) and version $B$ at time 2 (T2). Domain scores have a standardized mean of 100 and a standard deviation of 15 .

Brief Illness Perception Questionnaire (Broadbent et al. 2006). This measure consists of nine items, eight of which are based on a Likert scale ranging from 0 to 10 . The eight items include 'How much does your illness affect your life?' ; 'How long do you think your illness will continue?'; 'How much control do you feel you have over your illness?'; 'How much do you think your treatment can help your illness?'; 'How much do you experience symptoms from your illness?'; 'How concerned are you about your illness?'; 'How well do you feel you understand your illness?'; and 'How much does your illness affect you emotionally?' The Likert scale indicates perceived severity of illness experience. For example, 'How much does your illness affect your life?' $(0=$ no affect at all, $10=$ severely affects my life). The greater the score, the more severe the participant perceives their condition to be. The final item asks participants to list in rank order the three most important factors that they believe caused their illness. This short, single-item measure was selected to reduce the measurement load on participants (multiitem measures tend to be lengthy).

Hospital Anxiety and Depression Scale (HADS; Zigmund $\mathcal{E}$ Snaith, 1983). This is a self-report measure developed to measure current anxiety and depression in medical settings. Cut-off scores are provided for borderline and possibly clinically significant scores. Participants identify how often they experience feelings of depression or anxiety (or both). Fourteen statements are made such as 'I feel tense or wound up' and the participant has to rate how often they experience such feelings (e.g. most of the time through to not at all). Scores of $0-7$ are defined as being within the normal range, scores of 6-10 within the borderline range, and scores of $\geqslant 11$ are defined as being potentially clinically significant. For this sample the coefficient $\alpha$ 's were as follows: anxiety 0.80 , depression 0.79 , HADS total 0.89 .

Rosenberg Self-Esteem Scale (Rosenberg, 1989). This selfreport measures takes a Likert-scale format with 10 statements (e.g. 'I feel I have a number of good qualities') answered on a four-point scale. The scale ranges from 'strongly agree' to 'strongly disagree'. The greater the score, the greater the self-esteem an individual possesses. The score range is therefore between 0 and 40. For this sample the coefficient $\alpha$ was 0.88 . 
World Health Organization Quality of Life Measure - Brief Form (WHOQoL-BREF; WHO, 1996). This questionnaire comprises 26 questions relating to four specific life domains (physical, psychological, social and environmental). Typical questions include 'How would you rate your quality of life?' Participants have to rate each question on a five-point scale, where $1=$ very poor and $5=$ very good. Domain scores range from 0 to 20. In this sample the coefficient $\alpha$ 's were: physical 0.86 , psychological 0.80 , social 0.68 , environment 0.79 .

Patient Generated Index (Ruta et al. 1994). This is an alternative QoL measure that allows the participant to choose which areas of their life have been most affected by their illness. Each participant identifies the five most important areas of their life. They are then asked to score each of the five important areas with regard to how badly these areas have been affected by their illness $(0=$ the worst you could imagine and $10=$ exactly as you would like to be). The participants must also score their general health and non-health-related areas of their lives on this scale. Finally, the participant is given 14 'imaginary points' that they can spend improving the seven areas of their life. Participants give the highest number of points to the area they would like to improve most. For example, they may give all 14 points to one of the seven areas and no points to the other six areas. Alternatively, they may distribute the points equally among the seven available areas. The total score varies from 0 to 100 .

The entire procedure was then repeated approximately 4 to 5 weeks later. Patients with both CFS and AIFD often report inconsistency in their neuropsychological symptoms and, by repeating the assessments at a later date, the authors hoped to increase the reliability of the data and test consistency of results over time.

\section{Results}

\section{Power calculation and statistical analysis}

In this exploratory study we aimed to test for a medium effect size (0.4) with an $\alpha$ set at 0.05 with a power of 0.80 . We carried out a G-power calculation for a repeated-measures design with group factor (three levels) and within-subject factor (time) at two levels, and we based the power calculation on the betweenfactors (group) effect. This indicated that we required a sample size of 22 in each of the three groups. We tested for main effects of group and group $\times$ time interactions using 3 (group) $\times 2$ (time) analysis of variance (ANOVA), followed up by Bonferroni post-hoc comparisons. Categorical data were tested using $\chi^{2}$. Associations between variables were tested using
Pearson's correlations. All analyses were computed using SPSS version 16 (SPSS Inc., Chicago, IL, USA).

\section{Description of participants}

To test whether the three groups were well matched in terms of age, IQ and education level, a series of oneway ANOVAs and $\chi^{2}$ comparisons were conducted. The three groups were well matched in terms of age, IQ and education but were significantly different in terms of gender $\left(\chi^{2}=7.806, p=0.03\right)$. However, gender was found only to be related to HADS scores, and was only included as a covariate when analysing between group differences in HADS scores. For SES, participants were graded on the basis of their postcodes using the ACORN Geodemographic Classification Tool (www.caci.co.uk/acorn/acornmap.asp). Participants were identified as being inclusive in one of five categories $(1=$ wealthy achievers, $2=$ urban prosperity, $3=$ comfortably off, $4=$ moderate means, $5=$ hard pressed). We collapsed sociodemographic categories 1 and 2 and 4 and 5 and tested for betweengroup differences using $\chi^{2}$. No significant differences were found between the groups for SES $\left(\chi^{2}=3.070\right.$, $p=0.55)$.

\section{Results of analyses}

For the majority of the analyses, no significant group $x$ time interactions were observed, therefore main effects (mean scores across the two time points) are presented.

\section{Neuropsychological status}

A series of repeated-measures ANOVAs were conducted $(d f=2,59)$. Significant between-group differences were found between the groups on the domains of immediate memory, visuo-constructional tasks, attention and delayed memory (see Table 1). Bonferroni post-hoc analyses showed that the CFS group were significantly more impaired than the healthy participants on tests of immediate, delayed memory and visuo-constructional tasks. The CFS group were also significantly more impaired than the AITD group on tests of attention. Although there were no significant differences between the groups on the language task, there was a significant group $\times$ time interaction, the CFS group improved slightly, the AITD deteriorated slightly and the healthy participants remained fairly consistent over time: CFS mean $\mathrm{T} 1=92.5, \mathrm{~T} 2=97.1$, AITD T1 $=107.0, \mathrm{~T} 2=96.8$, controls $\mathrm{T} 1=100.3, \mathrm{~T} 2=$ $101.3\left[F(2,59)=9.42, p<0.001, \eta_{\mathrm{p}}^{2}=0.24\right]$.

In summary, the main result observed was that CFS patients were consistently more impaired on tests of 
Table 1. Between-group comparisons of neuropsychological status, mood quality of life and self-esteem

\begin{tabular}{|c|c|c|c|c|c|c|c|}
\hline & CFS & AITD & Control & $F$ & $p$ & $\eta_{\mathrm{p}}^{2}$ & Post-hoc \\
\hline \multicolumn{8}{|l|}{ RBANS } \\
\hline Immediate memory & $88.8(2.9)$ & $96.4(3.1)$ & $104.4(3.0)$ & 6.77 & 0.002 & 0.19 & $\mathrm{CFS}<\mathrm{C}$ \\
\hline Visuospatial/constructional ability & $92.9(2.7)$ & $95.5(2.8)$ & $104.1(2.7)$ & 4.71 & 0.013 & 0.14 & $\mathrm{CFS}<\mathrm{C}$ \\
\hline Language & $94.8(2.1)$ & $101.9(2.2)$ & $100.8(2.1)$ & 3.01 & 0.06 & 0.09 & \\
\hline Attention & $89.7(3.4)$ & $103.1(3.5)$ & $98.5(3.4)$ & 4.00 & 0.023 & 0.12 & $\mathrm{CFS}<\mathrm{AITD}$ \\
\hline Delayed memory & $87.2(2.9)$ & $93.5(3.0)$ & $101.0(3.0)$ & 5.44 & 0.007 & 0.16 & $\mathrm{CFS}<\mathrm{C}$ \\
\hline \multicolumn{8}{|l|}{ AFFECT } \\
\hline HADS Anxiety & $10.8(0.9)$ & $9.8(0.9)$ & $7.1(0.9)$ & 4.92 & 0.01 & 0.15 & $\mathrm{CFS}>\mathrm{C}$ \\
\hline HADS Depression & $10.4(0.8)$ & $7.6(0.9)$ & $3.9(0.8)$ & 15.5 & 0.001 & 0.35 & CFS, AITD $>$ C \\
\hline Self-esteem & $15.2(1.1)$ & $16.8(1.1)$ & $20.4(1.1)$ & 5.75 & 0.005 & 0.16 & $\mathrm{CFS}<\mathrm{C}$ \\
\hline \multicolumn{8}{|l|}{ WHOQoL } \\
\hline Physical & $9.6(0.5)$ & $12.4(0.5)$ & $16.1(0.5)$ & 47.7 & 0.001 & 0.62 & $\mathrm{CFS}<\mathrm{AITD}<\mathrm{C}$ \\
\hline Psychological & $11.4(0.5)$ & $12.4(0.5)$ & $14.6(0.5)$ & 9.69 & 0.001 & 0.25 & CFS, AITD $<$ C \\
\hline Social & $12.3(0.7)$ & $12.6(0.7)$ & $14.4(0.7)$ & 3.00 & 0.06 & 0.09 & \\
\hline Environment & $13.3(0.5)$ & $15.3(0.5)$ & $14.2(0.5)$ & 4.39 & 0.017 & 0.13 & CFS $<$ AITD \\
\hline PGI & $33.1(3.7)$ & $45.1(3.8)$ & $56.9(3.7)$ & 10.61 & 0.001 & 0.27 & $\mathrm{CFS}<\mathrm{C}$ \\
\hline
\end{tabular}

CFS, Chronic fatigue syndrome; AITD, autoimmune thyroid disease; C, control group; RBANS, Repeatable Battery for the Assessment of Neuropsychological Status; HADS, Hospital Anxiety and Depression Scale; WHOQoL, World Health Organization Quality of Life - Brief Form; PGI, Patient Generated Index.

Values given as mean (standard error).

immediate and delayed memory, visuo-constructional abilities and attention.

\section{Mood}

A series of repeated-measures ANOVAs were again conducted. Significant differences were found between the groups on measures of anxiety, depression and self-esteem and the results were consistent over time. Post-hoc analyses highlighted that the CFS and AITD groups had significantly higher self-reported levels of depression than the healthy participants. The CFS group had significantly higher levels of anxiety and lower self-esteem than the healthy participants. The results of the mood measures are shown in Table 1.

We then reanalysed the neuropsychological data controlling for mood. We did this because it is well established that depressed mood can impair neuropsychological performance (Cope et al. 1995; Marshall et al. 1997; Wearden \& Appleby, 1997). Therefore, the neuropsychological impairment we report above could be the result of mood, not CFS or AITD. We therefore computed composite total HADS anxiety/depression scores by taking an average of the T1 and T2 scores and entered this composite mood score as a covariate. When we reanalysed the data, all neuropsychological between-group differences were rendered nonsignificant, with the exception of attention: $C F S=91.4$
$($ S.E. $=3.6), \quad \mathrm{AITD}=103.6 \quad($ S.E. $=3.5), \quad$ healthy controls $=96.4 \quad(3.7) \quad\left[F(2,58)=3.17, p=0.049, \eta_{\mathrm{p}}=0.10\right.$, CFS $<$ AITD]. The effect of time and interaction of group $\times$ time was not significant. Thus the important finding is that, even after controlling for mood, the CFS group had significantly greater impairment in attention than the AITD clinical comparison group.

\section{Quality of life}

Once more, a series of repeated-measures ANOVAs were conducted. Significant differences were found between the groups in physical, psychological and environmental WHOQoL domains, and also on the Patient Generated Index (PGI) measure. Post-hoc analyses highlighted that the CFS group had significantly lower self-reported QoL in the physical and psychological QoL domains than both the AITD and healthy participants. The CFS group also scored lower on Environment QoL than the AITD group and lower than the healthy participants on the PGI. The differences were largely consistent over time, but the CFS group demonstrated a slight deterioration in physical QoL between T1 and T2.

\section{Illness perceptions}

A series of repeated-measures ANOVAs were run comparing the illness perceptions of the AITD and CFS groups. No group $\times$ time interactions were found 
but main group effects $(\mathrm{df}=1,39)$ were observed for timeline $[\mathrm{CFS}=7.3$ (S.E. $=0.4$ ), $\mathrm{AITD}=9.6$ (S.E. $=0.4$ ), $\left.F=14.8, p=0.001, \eta_{\mathrm{p}}^{2}=0.27\right]$, treatment control [CFS = 3.1 (s.E. $=0.6$ ), $\mathrm{AITD}=7.1$ (s.E. $=0.6$ ) $, F=23.2, p=0.001$, $\left.\eta_{\mathrm{p}}^{2}=0.37\right]$ and concerns $[\mathrm{CFS}=8.0($ s.E. $=0.5), \mathrm{AITD}=$ 6.1 (S.E. $\left.=0.5), F=6.82, p=0.013, \eta_{\mathrm{p}}^{2}=0.15\right]$. Thus, the AITD group believed that their condition would last longer, that they had greater treatment control over their condition, and expressed less concern over their condition compared with the CFS group. We then reanalysed the illness perception data controlling for mood using the composite anxiety and depression scores. The results showed that the AITD group's perception of their condition having a longer timeline and of having more treatment control and concern was not accountable by mood and remained significant.

In terms of attributing cause, the following main reasons were suggested: flu/virus $(\mathrm{CFS}=10, \mathrm{AITD}=$ $1)$, weakened immune system $(\mathrm{CFS}=2, \mathrm{AITD}=0)$, stress $(\mathrm{CFS}=5, \mathrm{AITD}=1)$, genetic cause $(\mathrm{CFS}=3$, $\mathrm{AITD}=6)$, not specified $(\mathrm{CFS}=3, \mathrm{AITD}=10)$.

\section{Antibody status}

Finally, we conducted a series of correlations to determine the relationship between antibody status in the AITD group and neuropsychological impairment, mood and QoL. All correlations proved to be nonsignificant.

\section{Discussion}

This study is the first to assess neuropsychological impairment longitudinally in a group of individuals with CFS and to use a comparison group of individuals with AITD, in addition to a healthy control group.

The first main finding of this study highlighted that the CFS group were significantly more impaired than the AITD group on attention. This finding therefore supports those of other authors who have also provided objective evidence for poor attention within the CFS population (McDonald et al. 1993; Ray et al. 1993; Sandman et al. 1993; Cope et al. 1995; Marshall et al. 1997; Johnson et al. 1998). The impairment the CFS group showed in attention was not accounted for by differences in mood. This contradicts the findings of previous authors who have claimed that cognitive complaints in CFS are specifically secondary to depressed or anxious mood (Smith, 1991; Grafman et al. 1993; McDonald et al. 1993). These results suggest that the core neuropsychological deficit in CFS is attentional, and that other cognitive deficits that have been reported may be secondary to this.
Turning to consider QoL, the CFS group reported lower QoL in the physical and psychological domains than both the AITD and healthy participants. Poor QoL has been reported in CFS populations worldwide (Hardt et al. 2001). Anderson \& Ferrans (1997) used a mixed-method approach to QoL in CFS patients. The results from the interviews highlighted a devastating effect on former social relationships, including relationship strain and subsequent loss of relationships. An inability to engage in social activities, economic stain, loss of purpose, self-worth, identity and selfesteem were all consequences of the onset of the condition. Given these qualitative findings, it is perhaps not surprising that the CFS group in our study had lower overall QoL than the AITD group. The AITD group were receiving treatment for their condition that helped them to manage the physical symptomatology of their condition. This was not true for the CFS group, many of whom were receiving no treatment for the physical symptoms of their condition. Given that many of the CFS participants were no longer able to work, many participants reported having to move house and experienced secondary economic strain. In addition to chronic fatigue, many members of the CFS group also reported chronic pain and, as a result, many experienced difficulty in going out. This again might account for their lower self-reported QoL in the physical and psychological domains.

We now turn to illness perceptions. In the present study, the AITD group believed that their condition would last longer but that they had more treatment control over their condition, and that they had less concern regarding their condition than the CFS group. This is perhaps not surprising given that AITD is a lifelong condition and the majority of the AITD participants were receiving treatment at the time of assessment. Many more participants in the CFS than the AITD group attributed their conditions to a viral aetiology, thus supporting Moss-Morris \& Chalder's (2003) findings (compared to their RA control group, the CFS group were more likely to attribute cause to a viral aetiology or immune system dysfunction). The CFS group were also more likely to consider stress as a cause, and overall, were more likely than the AITD group to attribute a cause for their condition (50\% of the AITD group failed to identify a cause). It is striking that the majority of CFS participants attributed a viral origin in light of the proposal by Richman et al. (2002) that Western medicine's failure to identify a viral aetiology for CFS has promoted a paradigmatic shift in research perspectives; it is possible that this shift underlies the tendency of health professionals to focus on sociocultural and psychiatric explanations for CFS (Ware, 1992; Cooper, 1997; Chaudhuri \& Behan, 2004; Dickson et al. 2007). For many CFS patients, this 
increases the risk that people with the condition may be viewed as being 'malingerers' (Ware, 1992) or as attempting to escape burdening roles (cf. Parson's 1951 description of the 'sick role'). Given these findings, it is perhaps not surprising then that the CFS participants (1) were keen to attribute cause for their condition and (2) advocated a physiological aetiology for that condition.

Edwards et al. (2001) found that individuals with CFS who had mood disturbance and greater levels of fatigue also demonstrated more 'catastrophic thinking'. Ray et al. (1992) propose that disturbed mood can directly affect CFS by exacerbating symptoms and amplifying the illness experience. We propose the opposite may also be true: concern about one's health (as found in the present study) may exacerbate CFS symptomatology and in turn lead to greater anxiety and depression. More specifically, patients who are particularly symptom focused or overly concerned may use a proportion of their attentional resources focusing on symptoms, thus making competing complex attentional tasks more difficult. Further research to clarify the causal direction of the relationship between symptom focus, affective status and attention is warranted.

A practical implication of the key finding of a core attentional deficit in CFS relates to treatment. It is likely that CFS patients may have difficulty attending to complex material in cognitive behavioural therapy (CBT) interventions with an increased likelihood of forgetting important components. We propose that such patients would be helped by consolidating material using additional handouts or worksheets, for example.

\section{Limitations}

We conclude with a consideration of the limitations of the current study. First, the CFS participants in this study may differ from those in other studies in that they did not have a prior history of affective disorder or other psychiatric conditions. Second, it is important to acknowledge that the sample sizes were relatively small. The voluntary nature of recruitment in both the CFS and AITD groups will inevitably have introduced a selection bias. Third, this was a longitudinal study over a relatively short time-frame and no inferences can be made regarding the causal nature of neuropsychological impairment, mood, QoL and illness perceptions. Nevertheless, the findings are important in identifying, for the first time, attention as the key neuropsychological impairment in CFS, which appears to remain consistent over time and is not a consequence of affective status. We propose that other studies reporting deficits in other areas of cognition in
CFS (e.g. memory, psychomotor function) may reflect a secondary consequence of a core attentional deficit. Future research should investigate the psychosocial impact of the attentional impairment and its relationship to mood, illness perceptions, QoL and response to treatment.

\section{Acknowledgements}

We thank all of the participants for their time, and David Dall (West Lothian CFS Support Group), Liz Blackadder (Edinburgh MESH) and Jennifer Frame (Glasgow Central College of Commerce) for their cooperation. This research was conducted while the first author was working as a Research Fellow at the University of Stirling.

\section{Declaration of Interest}

None.

\section{References}

Abbey SE, Garfinkel PE (1990). Chronic fatigue syndrome and the psychiatrist. Canadian Journal of Psychiatry 35, 625-626.

Anderson JJ, Ferrans CE (1997). The quality of life of persons with chronic fatigue syndrome. Journal of Nervous Mental Disorders 185, 359-365.

Ax S, Gregg V, Jones D (2001). Coping and illness cognitions: chronic fatigue syndrome. Clinical Psychology Review 21, 161-182.

Broadbent E, Petrie KJ, Main J, Weinman J (2006). The Brief Illness Perception Questionnaire. Journal of Psychosomatic Research 60, 631-637.

Busichio K, Tiersky LA, DeLuca J, Natelson BH (2004). Neuropsychological deficits in patients with chronic fatigue syndrome. Journal of the International Neuropsychological Society 10, 278-285.

Campion P, Bertie C, Chalder T, Cox D, Fleming C, Lant A, MacIntyre A, Moore M, Patterson D, Pearson V, Pheby D, Sweeney N, Skyes R, Weir W; The National Task Force on CFS/ME (1998). NHS Services for People with Chronic Fatigue Syndrome/Myalgic Encephalomyelitis. Westcare: Bristol.

Chalder T, Deale A, Wessely S (1999). Management of chronic fatigue syndrome. In Difficult Clinical Problems in Psychiatry (ed. M. Lader and D. Naber), pp. 135-153. Martin Dunitz: London.

Chaudhuri A, Behan PO (2004). Fatigue in neurological disorders. Lancet 363, 978-988.

Christodoulou C, DeLuca J, Lange G, Johnson SK, Sisto SA, Korn L, Natelson BH (1998). Relation between neuropsychological impairment and functional disability in patients with chronic fatigue syndrome. Journal of Neurology, Neurosurgery and Psychiatry 64, 431-434. 
Cooper L (1997). Myalgic encephalomyelitis and the medical encounters. Sociology of Health and Illness 90, 17-37.

Cope H, Mann A, Pelosi A, David A (1996). Psychosocial risk factors for chronic fatigue and chronic fatigue syndrome following presumed viral illness: a case-control study. Psychological Medicine 26, 1197-1209.

Cope H, Pernet A, Kendall B, David A (1995). Cognitive functioning and magnetic resonance imaging in chronic fatigue. British Journal of Psychiatry 167, 593-597.

David AS (1991). The post-viral fatigue syndrome and psychiatry. British Medical Bulletin 47, 966-988.

DeLuca J (1995). Neuropsychological impairments in chronic fatigue syndrome, multiple sclerosis and depression. Journal of Neurology, Neurosurgery and Psychiatry 28, 38-43.

DeLuca J, Christodoulou C, Diamond BJ, Rosenstein ED, Kramer N, Natelson BH (2004). Working memory deficits in chronic fatigue syndrome: differentiating between speed and accuracy of information processing. Journal of the International Neuropsychological Society 10, 101-109.

DeLuca J, Johnson SK, Beldowicz D, Natelson BH (1995). Neuropsychological impairment in chronic fatigue syndrome, multiple sclerosis and depression. Journal of Neurology, Neurosurgery and Psychiatry 56, 684-689.

Dickson A, Knussen C, Flowers, P (2007). Stigma and the delegitimation experience: an interpretative phenomenological analysis of people living with chronic fatigue syndrome. Psychology and Health 22, 851-867.

Edwards R, Suresh R, Lynch S, Clarkson P, Stanley P (2001). Illness perceptions and mood in chronic fatigue syndrome. Journal of Psychosomatic Research 50, 65-68.

Fukuda K, Straus S, Hickie I, Sharpe M, Dobbins J, Komaroff A (1994). The chronic fatigue syndrome: a comprehensive approach to its definition and study. Annals of Internal Medicine 121, 953-959.

Grafman J, Schwatz V, Dale JK, Scheffers M, Houser C, Straus SE (1993). Analysis of neuropsychological functioning in patients with chronic fatigue syndrome. Journal of Neurology, Neurosurgery and Psychiatry 56, 684-689.

Hardt J, Buschwald D, Wilks D, Sharpe M, Nix WA, Egle UT (2001). Health-related quality of life in patients with chronic fatigue syndrome: an international study. Journal of Psychosomatic Research 51, 431-434.

Hickie I, Lloyd A, Wakefield D, Parker G (1990). The psychiatric status of patients with chronic fatigue syndrome. British Journal of Psychiatry 156, 534-540.

Johnson SK, DeLuca J, Diamond BJ, Natelson BH (1998). Memory dysfunctioning in fatiguing illness: examining interference and distraction in short-term memory. Cognitive Neuropsychiatry 3, 269-285.

Johnson SK, DeLuca J, Natelson BH (1996). Depression in fatiguing illness: comparing patients with chronic fatigue syndrome, multiple sclerosis and depression. Journal of Affective Disorders 39, 21-30.

Katon WJ, Buchwald DS, Simon GE, Russo JE, Mease PJ (1991). Psychiatric illness in patients with chronic fatigue and those with rheumatoid arthritis. Journal of General Internal Medicine 6, 277-285.
Komaroff AL (1993). Clinical presentation of chronic fatigue syndrome. Ciba Foundation Symposium 173, 43-61.

Komaroff AL, Buchwald D (1991). Symptoms and signs of chronic fatigue syndrome. Revised Infectious Diseases 13, S8-S11.

Krupp LB, Sliwinski M, Masur DM, Friedberg F, Coyle PK (1994). Cognitive functioning and depression in patients with chronic fatigue syndrome and multiple sclerosis. Archives of Neurology 51, 705-710.

Lange G, Steffener J, Cook DB, Bly BM, Christodoulou C, Liu WC, DeLuca J, Natelson BH (2005). Objective evidence of cognitive complaints in chronic fatigue syndrome: a BOLD fMRI study of verbal working memory. Neuroimage 26, 513-524.

Leventhal H, Nerenz D, Steele DJ (1989). Illness representations and coping with health threats. In Handbook of Psychology and Health (ed. A. Baum, S. E. Taylor and J. E. Singer), pp. 219-252. Erlbaum: Hillsdale, NJ.

MacDonald KL, Osterholm MT, LeDell C, Persing DH, Johnson RC, Barker JM, Peterson PK (1996). A casecontrol study to assess possible triggers and co-factors in chronic fatigue syndrome. American Journal of Medicine 100, 548-554.

Manu P, Lane TJ, Matthews DA (1998). Somatization disorder in patients with chronic fatigue. Psychosomatics $\mathbf{3 0}$, 388-395.

Marshall PS, Forstot M, Callies A, Peterson PK, Schenck CH (1997). Cognitive slowing and working memory difficulties in chronic fatigue syndrome. Psychosomatic Medicine 59, 58-66.

McDonald E, Cope H, David A (1993). Cognitive impairment in patients with chronic fatigue syndrome: a preliminary study. Journal of Neurology, Neurosurgery and Psychiatry 56, 812-815.

Michiels V, de Gucht V, Cludyts R, Fischler B (1999). Attention and information processing efficiency in patients with chronic fatigue syndrome. Journal of Clinical and Experimental Neuropsychology 21, 709-729.

Moss-Morris R, Chalder T (2003). Illness perceptions and levels of disability in patients with chronic fatigue syndrome and rheumatoid arthritis. Journal of Psychosomatic Research 22, 305-308.

Moss-Morris R, Petrie K (2001). Discriminating between chronic fatigue syndrome and depression: a cognitive analysis. Psychological Medicine 31, 469-479.

Moss-Morris R, Petrie KJ, Large RG, Kydd RR (1996). Neuropsychological deficits in chronic fatigue syndrome: artifact or reality? Journal of Neurology, Neurosurgery and Psychiatry 60, 474-477.

Parsons T (1951). The Social System. The Free Press: New York.

Pepper C, Krupp L, Friedberg F, Doscher C, Coyle P (1993). A comparison of neuropsychiatric characteristics in chronic fatigue syndrome, multiple sclerosis and major depression. Journal of Neuropsychiatry and Clinical Neurosciences 5, 200-205.

Petrie K, Moss-Morris R, Weinman J (1995). The impact of catastrophic beliefs on functioning in chronic fatigue syndrome. Journal of Psychosomatic Research 39, 31-37. 
Pheby D (1999). An Overview of the Recent Research Literature. Discussion document prepared for the Working Group on Chronic Fatigue Syndrome/Myalgic Encephalomyelitis. University of the West of England: Bristol.

Rakib A, White PD, Pinching AJ, Hedge B, Newbery N, Fakhoury WK, Priebe $\mathbf{S}$ (2005). Subjective quality of life in patients with chronic fatigue syndrome. Quality of Life Research 14, 11-19.

Randolph C (2002). Repeatable Battery for the Assessment of Neuropsychological Status (RBANS). The Psychological Corporation: New York.

Ray C, Jefferies S, Weir WRC (1997). Coping and other predictors of outcome in chronic fatigue syndrome: a 1-year follow-up. Journal of Psychosomatic Research 43, 405-417.

Ray C, Philips L, Weir WRC (1993). Quality of attention in chronic fatigue syndrome: subjective reports of everyday attention and cognitive difficulty and performance on tasks of focussed attention. British Journal of Clinical Psychology 32, 357-364.

Ray C, Weir WRC, Cullen S, Phillips S (1992). Illness perception and symptom components in chronic fatigue syndrome. Journal of Psychosomatic Research 36, 243-256.

Richman JA, Jason LA, Taylor RR, Hahn SC (2002). Feminist perspectives on the social construction of illness states. Health Care for Women International 22, 173-185.

Rosenberg M (1989). Society and the Adolescent Self-Image, revised edition. Wesleyan University Press: Middletown, CT.

Ross S, Fantie B, Straus SF, Grafman J (2001). Divided attention deficits in patients with chronic fatigue syndrome. Applied Neuropsychology 8, 4-11.

Ruta D, Garratt A, Leng M, Russell I, McDonald L (1994). A new approach to the measurement of quality of life: the Patient Generated Index. Medical Care 32, 1109-1126.

Sandman CA, Barron JL, Nackoul K, Goldstein J, Fidler F (1993). Memory deficits associated with chronic fatigue immune dysfunction syndrome. Biological Psychiatry 33, 618-623.
Schweitzer R, Kelly B, Foran A, Terry D, Whiting J (1995). Quality of life in chronic fatigue syndrome. Social Science and Medicine 41, 1367-1372.

Smith A (1991). Cognitive changes in myalgic encephalomyelitis. In Post-viral Fatigue Syndrome (ed. R. Jenkins and J. Mowbray), pp. 179-204. John Wiley \& Sons: Chichester.

Surawy C, Hackman A, Hawton K, Sharpe M (1995). Chronic fatigue syndrome: a cognitive approach. Behavioural Research Therapy 33, 535-544.

van der Linden G, Chalder T, Hickie I, Koschera A, Sham P, Wessely S (1999). Fatigue and psychiatric disorder: different or the same? Psychological Medicine 29, 863-868.

Van Hoof E, Cluydts R, De Meirleir K (2003). Atopic depression as a secondary symptom in chronic fatigue syndrome. Medical Hypotheses 61, 52-55.

Ware NC (1992). Suffering and the social construction of illness: more questions than answers. Journal of Epidemiology and Community Health 46, 92-97.

Wearden A, Appleby L (1997). Cognitive performance and complaints of cognitive impairment in chronic fatigue syndrome. Psychological Medicine 27, 81-90.

Wechsler D (1981). Wechsler Adult Intelligence Scale - Revised. The Psychological Corporation: New York.

Wessely S, Nimnuan C, Sharpe M (1999). Functional somatic syndromes: one or many? Lancet 354, 936-939.

Wessely S, White PD (2004). There is only one functional somatic syndrome. British Journal of Psychiatry 185, 95-96.

WHO (1996). World Health Organization Quality of Life - Brief Form (WHOQoL-BREF). World Health Organization: Geneva.

Wood GC, Bentall RP, Gopfert M, Dewey ME, Edwards RHT (1994). The differential response of chronic fatigue, neurotic and muscular dystrophy patients to experimental psychological states. Psychological Medicine 24, 357-364.

Zigmund AS, Snaith RP (1983). Hospital Anxiety and Depression Scale (HADS). Acta Psychiatrica Scandinavica 67, 361-370. 